\title{
A COMPUTATIONAL APPROACH TO GENERATE DESIGN WITH SPECIFIC STYLE
}

\author{
Wang, Da (1); \\ Li, Jiaqi (1); \\ Ge, Zhen (2,3); \\ Han, Ji (1) \\ 1: University of Liverpool; \\ 2: University of Dundee; \\ 3: University of Technology Sydney
}

\begin{abstract}
Creativity is crucial in design. In recent years, growing computational methods are applied to improve the creativity of design. This paper aims to explore an approach to generate creative design images with specific feature or design style. A Generative Adversarial Network model is applied in the approach to learn the specific design style. The target products will be projected into the latent space of model to transfer their styles and generate images. The generated images combine the features of the specific design style and the features of the target product. In the experiment, the approach using the generated images to inspire the human designer to generate the creative design in according styles. According to the primary verification by participants, the generated images can bring novelty and surprise to participants, which gain the positive impact on human creativity.
\end{abstract}

Keywords: Artificial intelligence, Creativity, Computational design methods

\author{
Contact: \\ Wang, Da \\ University of Liverpool \\ Industrial Design \\ United Kingdom \\ da.wang@liverpool.ac.uk
}




\section{INTRODUCTION}

In design, creativity is an ability to produce novel (original, unexpected) and appropriate (useful, relevant) works (Sternberg and Lubart, 1999). It is crucial to designers, especially in idea generation. Conventional methods such as brainstorming (Osborn, 1967), mind mapping (Buzan, 1974), and TRIZ (Altshuller, 1984) have been widely used in design to enhance designers' creativity. In recent years, there is an emerging trend in using computational approaches to generate images of design in a direct manner. For example, generative design is a design method for exploring a new design by transferring the initial dataset to many potential design results ( $\mathrm{Li}$ and Lachmayer, 2018). Style transfer can compose design sketches with stylized images to generate new designs (Gatys et al., 2016; Huang et al., 2018). Using the generative adversarial network (GAN) model is another computational approach to automatically generate a design with preferences (Goodfellow et al., 2014).

However, most of the computational approaches do not involve human cognition to generate results. Design work is a comprehensive performance of multiple abilities (Bush, 1945). It is a decision making, exploration, and learning activity, which is purposeful and constrained (Gero, 1996). Factors such as technology, structure, material, cost, and policy need to be considered during the design process. Therefore, design tasks require good logical thinking to solve practical problems. Comparing with temporary computational approaches, human designers can gain better design results with the frame of these factors. It is vital for design to involve the consideration of human aspects (Vicente, 2013; Cooley, 2000). Combining human aspects and computational methods is a better choice in design. Using images generated by GAN to support designers in developing creative ideas is a typical example. For instance, Schmitt and Weiß (2018) proposed a trained GAN model that can independently generate multiple novel shapes of the chair, which are used to inspire designers to generate creative design sketches for new chair designs. This study agrees that using computational creativity to help human designers generate design ideas is a promising approach.

Human creativity is distinguished by Boden (2009) into three forms: exploratory creativity, transformational creativity and combinational creativity. In design, combinational creativity plays a significant role. It involves exploring creative combinations of concepts or ideas (Han et al., 2016; 2018a). There are many design methods such as design by analogy (Moreno et al. 2014; Han et al. 2018b) and bio-inspired design (Fu et al., 2014) that involves aspects of combinational creativity. Combinational creativity is also employed in computational approaches, for example Chen et al. (2019) came up with an advanced approach using a GAN model to produce combinational images to stimulate designers' creative minds. However, the GAN model needs to be pretrained by using product images in multiple categories to combine the features of those products in the final design. The process is time-consuming and could only produce low-quality images. If a new feature is tended to be included, the whole model needs to be retrained from the beginning.

This paper explores an approach that using the StyleGAN2 model to learn design style features and elements from selected images, which then transfers the design style learned to exist products. A primary survey is conducted among several designers to explore whether the outcomes produced by the approach are helpful for designers in generating design ideas. The advantage of the approach is that it could generate high-quality images in comparison with using solely style transfer. The model combines multiple features and only need to be trained once, which could then be applied to any exiting product images. This paper has provided new and useful insights on using artificial intelligence techniques to support designers in generating ideas.

\section{RELATED WORK}

\subsection{Computational methods to support design}

Computer-aided design (CAD) has been broadly used by designers. From the earliest CAD software Sketchpad (Sutherland, 1964) to the newest Autodesk software, the way of designing has changed significantly changed. Besides, designers can store electronic documents and share design documents with others by using CAD tools even at a far distance. In the 1980s, Computer-supported cooperative work (CSCW) (Grudin, 1994) was proposed. It aims to support users' collaboration activities by the computer system. Benefiting from the computer system and the advent of the computer network, designers started to collaborate with each other to efficiently accomplish common large design projects and even international design projects. 


\subsection{Computational methods for supporting design creativity}

There are many theoretical approaches to enhance human creativity. As illustrated in the previous section, human creativity involves exploratory, transformational and combinational creativity (Boden, 2009). Developing a knowledge base is recommended by many researchers. Hayes (1978) believes that abundant background knowledge allows the human mind to have large information storage space, which is beneficial for exploratory creativity, especially in science, literature, art and mathematics. The expert system (Jackson, 1998) can provide specific knowledge for the designer base on design projects, which is useful for enhancing explorational creativity. The high-performance Convolutional Neural Network was used to transfer image styles between arbitrary images (Gatys et al., 2016), which can help designers generate their transformational creativity. The use of combinational creativity in computational approaches has been explored by several researchers such as Han et al. (2016, 2018a). They came up with a tool, the Combinator, which can generate combinational textual and pictorial stimuli to support designers in creative idea generation.

\subsection{Computational creativity}

Methods in 2.2 are used to support designers in creative idea generation. However, there exist several approaches that could produce creative designs by themselves. For example, generative design can explore a new design by transferring the initial dataset to many potential design results ( $\mathrm{Li}$ and Lachmayer, 2018). In the conventional design process, human designers can gain inspiration by methods such as design by analogy and bio-inspired design. Computational methods such as Generative Adversarial Network (Goodfellow et al., 2014) are similar to these approaches, which can generate new sketches that borrow features from a sort of object in particular styles. For instance, DesignGAN is an approach for generating a shape-oriented bionic design that combines the features from a biological source domain and the shape of a design target (Yu et al., 2018). In this case, the human designer is indispensable for the design process to gain desirable results with preference from clients.

\subsection{The introduce of the Generative Adversarial Network (GAN)}

The Generative Adversarial Network is a well-known deep learning framework, which is appropriate to be applied in image generation. Generally, there are two neuron networks in a GAN framework, which are called the generator and the discriminator. The generator can generate images by random or provided noise while the discriminator can distinguish the real images and fake images. In the beginning, the generated After trained by a large number of images, these two models are well-trained adversarially to compete with each other, and their capabilities are improved. Finally, the discriminator cannot distinguish the fake images from the generator and real images. It means that the generator can generate convincing fake images (Goodfellow et al., 2014).

In this study, the aim of employing GAN is to produce high-resolution images (512*512 pixels) to interact with human designers. However, the conventional GAN could not produce high-resolution images because these images make it easy for the discriminator to distinguish if they are fake or real, which leads to an interrupt of the learning process. The StyleGAN model applies the strategy that gradually improves the image quality. In the beginning, the generator starts with low resolutions $(4 * 4$ pixels). Then, a new layer will be added when the training process converges (Karras et al., 2018). Eventually, this approach can help the network to generate high-resolution images. The SytleGAN2 model is applied in this study. It is an optimized version of StyleGAN, which can provide better image quality and reduce the training time (Karras et al., 2020).

\subsection{Latent space}

To generate fake images, the generator of StyleGAN2 requires unique seeds. In general, the seed is made from a pseudo random generator. This seed will be transferred into a vector as the input of the generator. In the training process, the generator learns to map the vector to features in the image (Shen et al, 2020).

The latent space is constructed by the vector. In general, it is represented by a multidimensional hyperspace. In the StyleGAN2 model, it is represented by a 512-dimensional hyper-sphere. The adjacent coordinates in latent space produce similar results, which is different from other models. It allows the user to modify an image by altering its coordinates of vector in latent space (Karras et al., 2020). 
Benefiting from the trait of the StyleGAN2 model, it is possible to convert the target image to a vector in the latent space. Then, the vector will be used to generate an image by StyleGAN2. In the beginning, the generated images contain the features of stylized design product image but are not similar to the target image. The method of interpolation will be applied and the feature of output images will be increasingly closer to the target image. After modification according to the difference between the generated images and target image, the generated images can be similar to the target image. In this process, the images generated contain the features of design product and target image.

\section{METHODOLOGY}

There are 3 processes in this approach. As shown in Figure 1, the training process is about training a StyleGAN2 model by specific design style images. Base on the random latent vector and noise, the generator can generate fake images. The loss function will help to modify the generator and discriminator according to the deviation between real images and generated image. One of the pretrained official StyleGAN2 models will be applied in the training process for better image quality and less training time. The model will be sequentially trained by stylized design product images. After the training process, the generator can produce images combining the features of cars and the chosen design style.

\section{Process 1: Training Process}

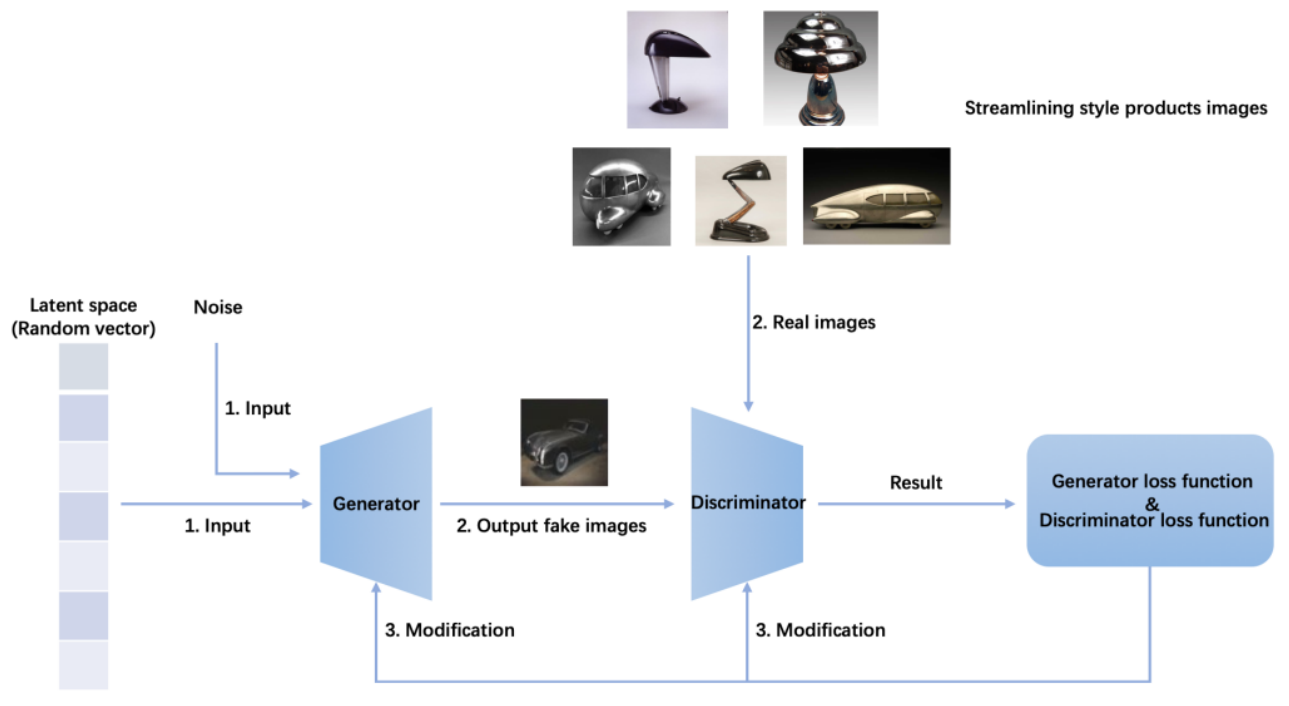

Figure 1. The training process of StyleGAN2 model

In Figure 2, the aim of this process is to project the real image to the latent space for style mixing. Firstly, the target image is encoded to a latent vector by the StyleGAN2 generator. Then a random vector is generated in latent space. This random vector will be modified to close the vector of real image step by step. In each step, there will be a generated image by the StyleGAN2 generator. In this case, the maximum step is 1000 because of the limitation of model. The output images reflect the shape variation from trained model to target product, which means these images combine the features of them. By this method, the design style can be applied to the target image. 


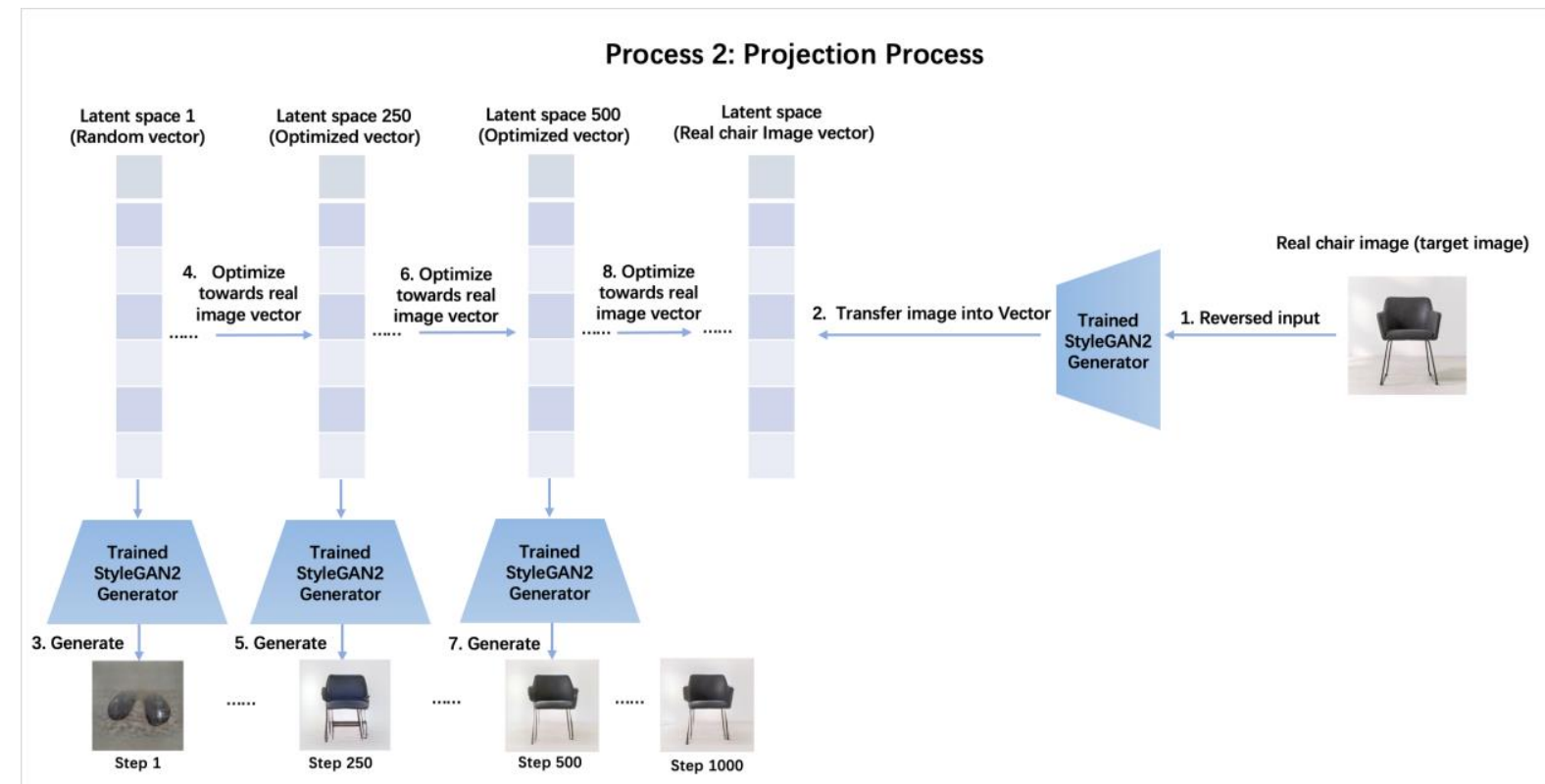

Figure 2. The projection process of StyleGAN2 model

In Figure 3, the generated image is used to inspire human designers to do their creative design. To ensure the validity of generated images and control the time of inspiration process, the output images are artificially selected to inspire designers. In selected images, the features of the real image should be retained because the design object should be clear for designer.

Process 3: Inspiration Process

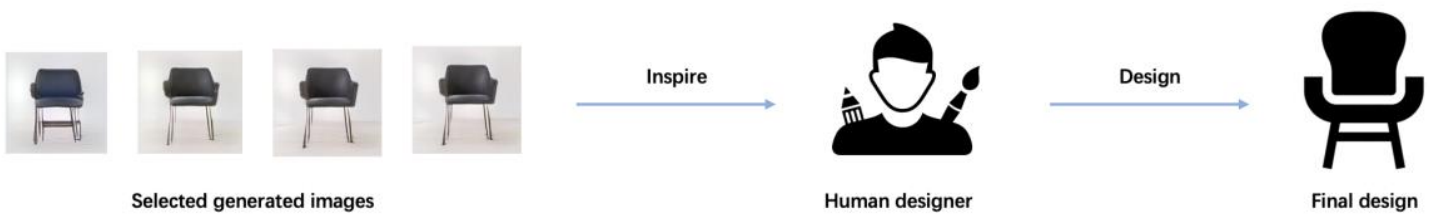

Figure 3. The inspiration process

\section{CASE STUDY}

In order to evaluate the approach, we have conducted a case study to apply the approach. The aim of the case study is to train a StyleGAN2 model that can generate stylized images based on the provided image. We selected streamlining-style products as the dataset because the feature of them are similar and easy to be learned by the model. Besides, we choose the official pretrained model that can generate high-quality car images as the base of our model because the target is close (most cars are in streamlining style). It will save a large amount of training time for the StyleGAN2 model. We choose images of the 2 chairs as the target image because this approach is suitable for transferring the objects in simple shape.

\subsection{Model Training}

Before the experiment, a StyleGAN2 model is trained by 1213 images of the streamlining-style product. For the best training result, the model was trained based on the official car model, which is good for generating streamlining-style objects. The model is trained for 5000 steps. In Figure 4, the 
images are generated base on random noise by trained StyleGAN2 model. These random generated contents are close to the official car model. At the same time, some features of streamlining design style have to be involved.

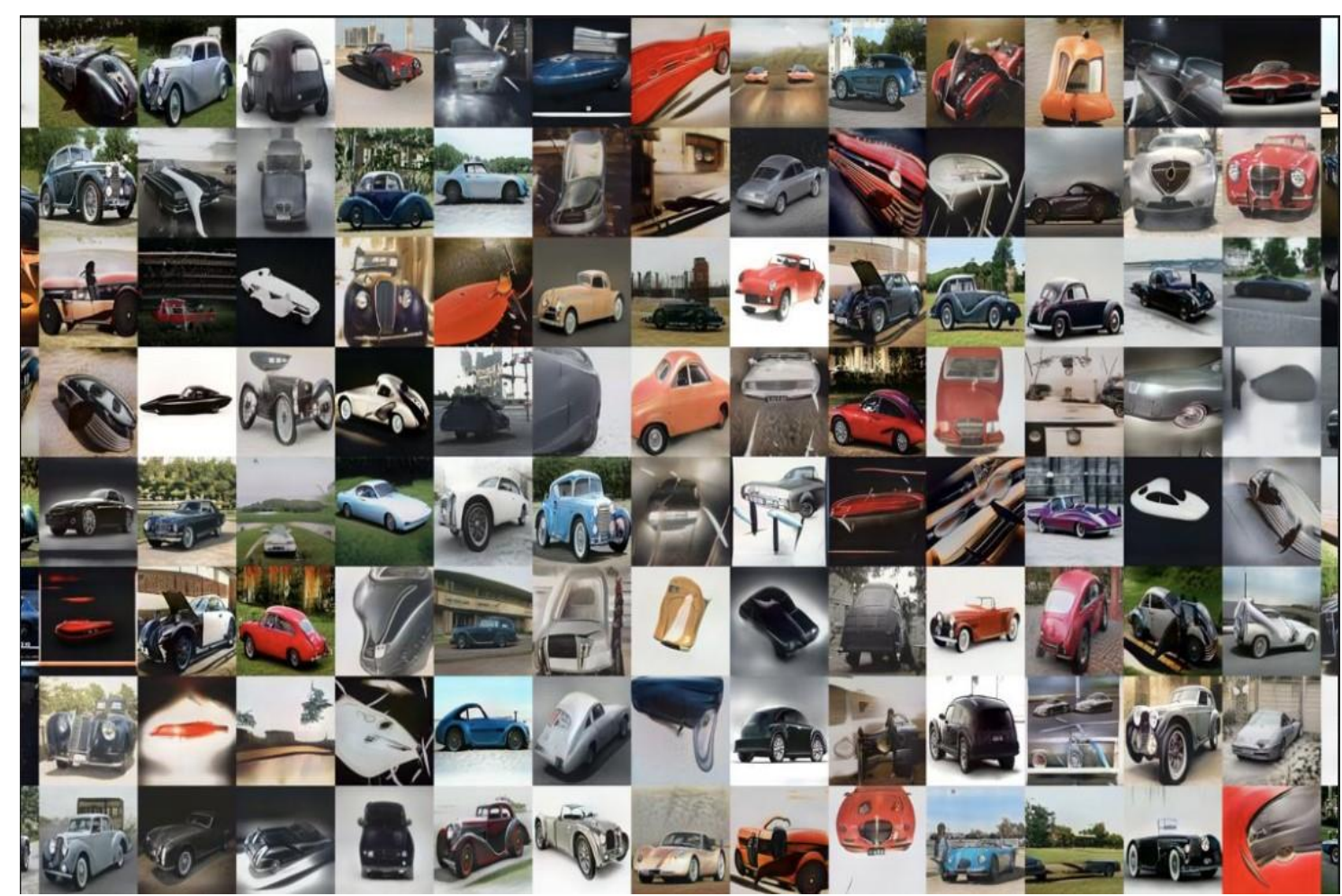

Figure 4. The random generated content of the trained model

\subsection{Case Study Result}

After training, the model can automatically generate images with features of streamlining design and car. In the latent space of the model, when random noise is provided, it gives a random latent vector, which can generate an image. When a chair image is provided, it will be encoded into a latent vector. Then, a random vector starts to be optimized towards the vector of real image. In this process, every time the random vector is modified, it will generate an image gains both the features of streamlining style and real chair image. The step means that the number of optimized times from the image generated by random latent vector to the latent vector of the real chair image.

Then, two chairs in different styles are chosen to transfer. The generated images of the two chairs by latent vector and the trained model in the 1st step, the 250th step, the 500th step, the 750th step and the 1000th step are shown in Figure 5. The shape of the chair is gradually changed in this process. Selecting the appropriate steps to stimulate the creativity of the designer is significant. It is because that if the original shape of the target image is different, the speed of variation is different accordingly. In the case study, the selected images should keep the features of streamlining style and the shape of provided chair. The appropriate generated images gain positive effect on inspiration. 


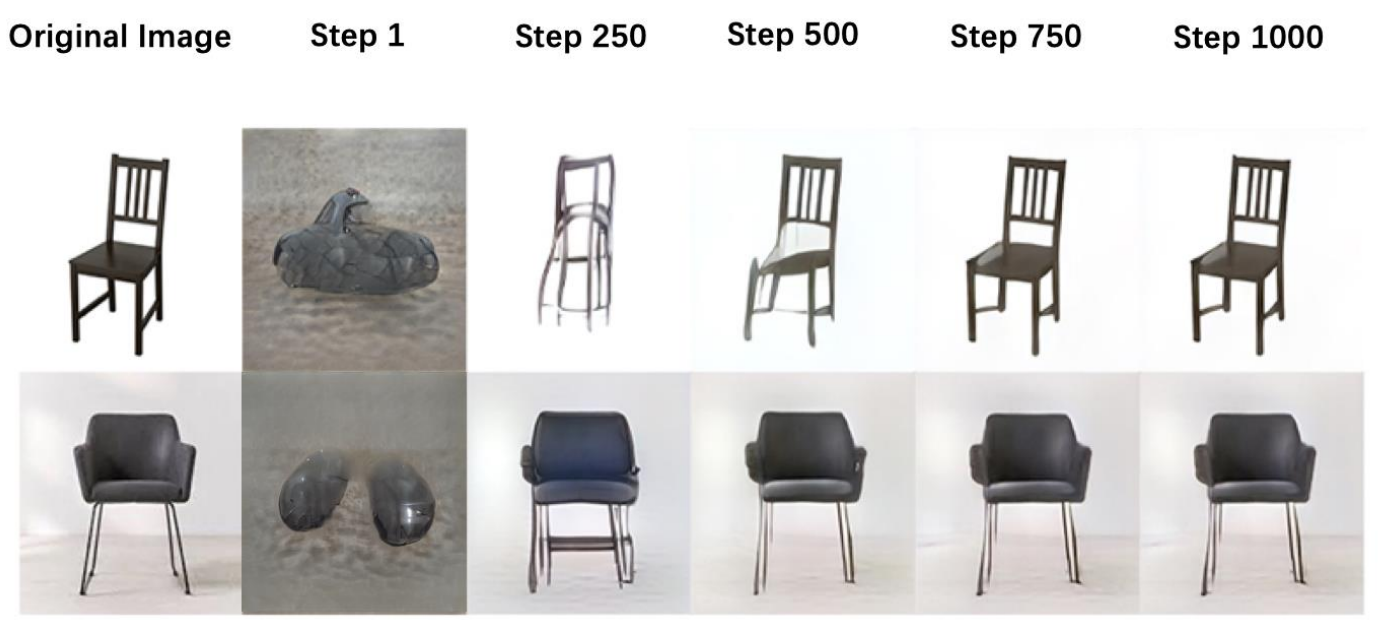

Figure 5. the projector of the real chair images in different steps

\subsection{Evaluation of the approach}

Six participants participated in the evaluation study. The age of them ranges from 28 to 33 . Four of them were design students, and two of them currently are professional designers.

The idea generation is a complex process in design. There are many aspects such as the way of interaction, work environment, design tools and reaction of designers that need to be researched. However, the face to face experiment is hard to implement in this period due to the pandemic. In the current stage, the experiment gains the interview and feedback of participants to research whether this approach can bring positive effect on human creativity in design. To simulate the environment of design and exclude external influence. Each of the participants must work alone during the experiment. They have limited time to think and answer questions after the inspiration process. To be more specific, 1) the participants are required to think about if they are asked to transfer the chair image into streamlining-style, what design sketch they will draw; and 2) from the step 250th to step 1000th of each chair, did they feel surprised (different from the sketches in their minds) and agree with that the images generated are potential design sketches of chairs, and from which step they start to have this feeling.

The interview results indicate that for the first chair, all participants have the feeling that the generated chairs inspired them from the 500th step. For the second chair, all participants have the surprise feeling from the 250th step. Five participants think that the images of the 750th step and the 1000th step have almost not changed. According to the feedback of participants, the generated images contain the potential to be stylized design sketches and inspire human designers.

\section{DISCUSSION}

The study is a primary exploration of using specific style image generator to stimulate the creativity of human designers and apply it to inspire designers. There are some advantages of this approach. Comparing with other conventional application of GAN in design area, this approach involves human efforts to gain the final design. The original idea of design is generated by StyleGAN2. Then, the human designer can be inspired by it and output the final design. The optimized StyleGAN2 model can generate high-quality images, which achieve a better effect on stimulating human designer by vision. Comparing with other style mixing model, this model is only needed to be trained once for multiple designs. Designers can apply the model on multiple targets by projecting their images into the model's latent space. The human factor plays an important role in the approach. Although generative algorithms can achieve high-quality results, it still requires the human designer to modify the details of design. In this approach, the generated images will be watched, considered, and redrawn by human designers. The designer can make sure that the output of the approach is logical and appropriate for the requirement of human users. 
However, there are some limitations in the case study. First, this model aims to learn the feature and style of a group of images. Therefore, if the generated images are too realistic, the projector may not gain the best result. According to the application of Generative Adversarial Network, training a model for a particular object cost plenty of time and data. For example, the official StyleGAN2 generative model that can automatically produce lifelike car images cost 57 million real car images to train. In this case, A pretrained StyleGAN2 official car model is applied to train the new streamlining-style model for saving time. However, it may lead to the consequence that there are too many features of car existed in the generator. This issue is considered in the selection of design style and design target of the experiment. To overcome wind resistance, streamlining is a common design style for the car. Benefiting from the official pretrained car model, the streamlining-style model can be trained by the limited streamlining style product image dataset and time. At present, the target of design must be a product with simple shape and clear usage. In this approach, the function of the target product should have a strong relationship with its appearance. Otherwise, the computational method cannot contribute to the function of design. Although the chair is a simple shape product, the performance of generated results is not good enough. Finally, the evaluation and verification of computational output are significant for the approach. In this study, human designers involved in a simple way. The complete process of using generated images to inspire designers is necessary. Besides, streamlining style may be not a typical enough design style for generating. It is chosen here because it is close to the style of pretrained model. Another reason is that streamlining style is widely used in product design, which means the final result can be evaluated better. The last issue is to explore whether this approach could really enhance the creativity of designers. In this study, there are only limited subjective interview with participants.

\section{FUTURE WORK}

First, if the GAN model is overtrained, the generated images will gain too many detailed features of the products in the training images rather than a design style. To avoid this situation of the StyleGAN2 model, a limitation of the training step should be set according to the final effect. The detailed training steps should be concluded according to multiple attempts of training. Then, the next stage study requires a single function target, which its shape can significantly influence the function. The GAN model can generate the appearance of the product. The model shows that it can inspire human designers. However, functionality is another significant element in creativity. Products such as bottle or handle, the shapes are related to their functionalities. Next, more design styles should be involved in the future. The style streamlining is wide, sometimes the output even close to other styles. Some unique enough design styles such as cyberpunk style and deconstructive style are required for generation and evaluation. Finally, the aim of the approach is to generate creative design output. Although the results in this study gain a positive impact on designers, the final outputs of human designers need to be evaluated.

In future research, the qualitative method will be used to explore the feeling of participants (Hammarberg et al., 2016). Interview, questionnaire and focus group will be considered to be used in the research. Participants will share their feelings about whether using the computational tool can enhance their creativity. However, it is challenging to interpret verbal, behavioural and observational data into numeric. Therefore, more evidence is needed to value the impact of the approach. Quantitative methods employ various statistical analysis methods to evaluate the results for exploring how designers perceive creativity. Quantitative methods such as Consensual Assessment Technique (CAT) (Baer and Kaufman, 2018; Amabile, 2018) will be applied to value the creativity of design. The sketches drawn in this approach can be compared with the design sketches generated under other stimulations in a similar situation. For example, the creativity of sketches could be compared with the creativity of participants' daily sketches on the same topic.

Due to the pandemic, the offline experiment is challenging to implement at present. An online experiment is planned to verify the result of approach. In addition to complete data collection, the interactions between human designers and computers or mobile devices is another vital issue. A user interface is planned to be designed to address the issue. 


\section{CONCLUSION}

The paper has proposed an approach that using the images generated by an optimized Generative Adversarial network to enhance the creativity of the human designer. Based on the relevant theory of combinational creativity, the generated images can combine the features of the specific design style and the target product, which is a benefit for improving human creativity. In the case study, a generator of streamlining style is trained. Base on the primary verification of participants, the generated images in some projection steps can bring novelty and surprise to participants, which gain positive impacts on human creativity. According to the analysis of the case study, some limitations of GAN model training and the process of evaluation are needed to be explored. There are still some additional tasks, such as filtering the generated images and evaluating the enhancement of participants' creativity, to be conducted in future research.

This study provides new inspirations for research of design methods or design tools. Although the exact improvement of idea generation is needed to be verified, the human designer has the potential to work with spontaneous creativity of machines. For research area of generative adversarial network, the study attempts to explore the potential workflow of human designer and artificial intelligence.

\section{ACKNOWLEDGEMENTS}

The author would like to acknowledge the Nvidia development team for the optimized StyleGAN2 model and the online pre-trained models, which are crucial for this paper.

\section{REFERENCES}

Altshuller, G. S. (1984) Creativity as an Exact Science: The Theory of the Solution of Inventive Problems. Gordon and Breach Publishers, http://dx.doi.org/10.1201/9781466593442

Amabile, T.M. (2018) Creativity in Context: Update to the social psychology of creativity, Westview, Boulder. http://dx.doi.org/10.4324/9780429501234-3

Baer, J. \& Kaufman, J.C., (2018) Assessing Creativity with the Consensual Assessment Technique, The Palgrave Handbook of Social Creativity Research, pp. 27-37. http://dx.doi.org/10.1007/978-3-319-954981_3

Boden, M.A. (2009) "Computer models of creativity", AI Magazine, Vol. 30, No. 3, pp. $23-23$.

Bush, V. (1945) "As we may think", Atlantic Monthly, Vol. 176, No. 1, pp. 101-108.

Buzan, T. (1974) Use your head, Rajpal \& Sons.

Chen, L., Shi, F., Han, J. and Childs, P.R. (2017) "A network-based computational model for creative knowledge discovery bridging human-computer interaction and data mining", in International Design Engineering Technical Conferences and Computers and Information in Engineering Conference, American Society of Mechanical Engineers. http://dx.doi.org/10.1115/detc2017-67228

Chen, L., Wang, P., Dong, H., Shi, F., Han, J., Guo, Y., Childs, P.R.N., Xiao, J. and Wu, C. (2019) "An artificial intelligence based data-driven approach for design ideation", Journal of Visual Communication and Image Representation, Vol. 61, pp. 10-22. http://doi.org/10.1016/j.jvcir.2019.02.009.

Chen, L., Wang, P., Shi, F., Han, J. and Childs, P. (2018) "A computational approach for combinational creativity in design", in DS 92: Proceedings of the DESIGN 2018 15th International Design Conference, pp. 1815-1824. http://dx.doi.org/10.21278/idc.2018.0375

Cooley, M. (2000) "Human-centered design", Information design, pp. 59-81.

Fu, K., Moreno, D., Yang, M. and Wood, K.L. (2014) "Bio-inspired design: an overview investigating open questions from the broader field of design-by-analogy", Journal of Mechanical Design, Vol. 136, No. 11. http://dx.doi.org/10.1115/1.4028289

Gero, J.S. (1996) "Creativity, emergence and evolution in design", Knowledge-Based Systems, Vol. 9, No. 7, pp. 435-448. http://dx.doi.org/10.1016/s0950-7051(96)01054-4

Gero, J.S. and Kannengiesser, U. (2004) "The situated function-behaviour-structure framework", Design Studies, Vol. 25, No, 4, pp. 373-391. https://doi.org/10.1016/j.destud.2003.10.010

Goodfellow, I., Pouget-Abadie, J., Mirza, M., Xu, B., Warde-Farley, D., Ozair, S., Courville, A. and Bengio, Y. (2014) "Generative adversarial nets", in Advances in neural information processing systems, pp. 2672-2680.

Grudin, J. (1994) "Computer-supported cooperative work: History and focus", Computer, Vol. 27, No. 5, pp. 1926. https://doi.org/10.1109/2.291294

Hammarberg, K., Kirkman, M., de Lacey S. (2016) "Qualitative research methods: when to use them and how to judge them", Human Reproduction, Vol. 31, No. 3, pp. 498-501. http://dx.doi.org/10.1093/humrep/dev334

Han, J., Shi, F., Chen, L. and Childs, P.R.N. (2018a) "The Combinator - a computer-based tool for creative idea generation based on a simulation approach", Design Science, 4, e11. http://dx.doi.org/10.1017/dsj.2018.7 
Han J, Shi F, Chen L, Childs, PRN (2018b). A computational tool for creative idea generation based on analogical reasoning and ontology. Artificial Intelligence for Engineering Design, Analysis and Manufacturing Vol. 32, pp. 462-477, https://doi.org/10.1017/S0890060418000082

Han, J., Shi, F. and Childs, P. (2016) "The Combinator: A computer-based tool for idea generation", in DS 84: Proceedings of the DESIGN 2016 14th International Design Conference, pp. 639-648. http://dx.doi.org/10. 1017/dsj.2018.7

Hayes, J.R. (1978) Cognitive psychology: Thinking and creating, Dorsey.

Huang, X., Liu, M.-Y., Belongie, S. and Kautz, J. (2018) "Multimodal unsupervised image-to-image translation", in Proceedings of the European Conference on Computer Vision (ECCV), pp. 172-189.

Jackson, P. (1998) Introduction to Expert Systems, Addison-Wesley Longman Publishing Co., Inc, Boston.

Karras, T., Laine, S., Aittala, M., Hellsten, J., Lehtinen, J. and Aila, T. (2020) "Analyzing and improving the image quality of stylegan", in Proceedings of the IEEE/CVF Conference on Computer Vision and Pattern Recognition, pp. 8110-8119. http://dx.doi.org/10.1109/cvpr42600.2020.00813

Karras, T., Aila, T., Laine, S., \& Lehtinen, J. (2018). "Progressive Growing of GANs for Improved Quality, Stability, and Variation" International Conference on Learning Representations 2018, CoRR.

Li, H. and Lachmayer, R. (2018) "Generative Design Approach for Modeling Creative Designs", in IOP Conference Series: Materials Science and Engineering, IOP Publishing, p. 012035. https://doi.org/10.1088/1757-899x/408/1/012035

Moreno, D.P., Hernandez, A.A., Yang, M.C., Otto, K.N., Hölttä-Otto, K., Linsey, J.S., Wood, K.L. and Linden, A. (2014) "Fundamental studies in Design-by-Analogy: A focus on domain-knowledge experts and applications to transactional design problems", Design Studies, Vol. 35, No. 3, pp. 232-272. http://dx.doi.org/10.1016/j.destud.2013.11.002

Osborn, A. (2012) Applied imagination-principles and procedures of creative writing, Read Books Ltd.

Schmitt, P. and Weiß, S. (2018) "The Chair Project: A Case-Study for using Generative Machine Learning as Automatism", 32nd Conference on Neural Information Processing Systems (NIPS 2018), Montréal, Canada.

Shen, Y., Gu, J., Tang, X., \& Zhou, B. (2020). "Interpreting the Latent Space of GANs for Semantic Face Editing", 2020 IEEE/CVF Conference on Computer Vision and Pattern Recognition (CVPR), pp. 92439252. http://dx.doi.org/10.1109/cvpr42600.2020.00926.

Sternberg, R.J. and Lubart, T.I. (1999) "The concept of creativity: Prospects and paradigms", Handbook of creativity, 1, pp. 3-15.

Sutherland, I. E. (1964). "Sketchpad a man-machine graphical communication system", Proceedings of the SHARE design automation workshop, Association for Computing Machinery, New York, Vol. 2, No. 5, R3. https://doi.org/10.1145/800265.810742

Vattam, S., Wiltgen, B., Helms, M., Goel, A.K. and Yen, J. (2011) "DANE: fostering creativity in and through biologically inspired design" in Design Creativity 2010, Springer, London, pp. 115-122. https://doi.org/10.1007/978-0-85729-224-7_16

Vicente, K.J. (2013) The Human Factor: Revolutionizing the Way People Live with Technology, Routledge.

Wu, J., Zhang, C., Xue, T., Freeman, B. and Tenenbaum, J. (2016) "Learning a probabilistic latent space of object shapes via 3d generative-adversarial modeling", Advances in neural information processing systems, Vol. 29, pp. 82-90.

Yu, S., Dong, H., Wang, P., Wu, C. and Guo, Y. (2018) "Generative Creativity: Adversarial Learning for Bionic Design", Artificial Neural Networks and Machine Learning - ICANN 2019: Image Processing, pp. 525536. http://dx.doi.org/10.1007/978-3-030-30508-6_42 